\title{
A motivação no Ensino de Ciências: análise de dez anos de trabalhos apresentados no ENPEC
}

\section{Wilson Carvalho \\ carvalhowilson87@gmail.com \\ orcid.org//0000-0003-0773-4894 \\ Universidade Estadual de Londrina (UEL) Londrina, Paraná, BR \\ Enio de Lorena Stanzani enio.stanzani@gmail.com Universidade Tecnológica Federal do Paraná (UTFPR), Apucarana, Paraná, BR \\ Marinez Meneghello Passos marinezmp@sercomtel.com.br orcid.org//0000-0001-8856-5521

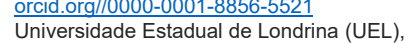 Londrina, Paraná, BR}

\section{RESUMO}

Este trabalho apresenta resultados de uma investigação sobre o conceito motivação a partir de trabalhos publicados no Encontro Nacional de Pesquisa em Educação em Ciências (ENPEC). Para primeira etapa analítica, foram analisados todos os trabalhos publicados nos anais das últimas seis edições do evento (2005 a 2015), totalizando 5693 artigos. Na sequência foram realizadas buscas pelo termo motivação, resultando em 93 trabalhos, os quais constituíram o corpus da presente pesquisa. Como ferramenta metodológica de análise e de coleta de dados, utilizouse a análise textual, com ênfase nos pressupostos da Análise de Conteúdo. A partir dos resultados, foi possível constatar que: no ENPEC os trabalhos relacionados ao conceito motivação não representam uma temática significativa dentre os temas pesquisados ao longo das últimas seis edições; o número de pesquisas que trazem o termo motivação com seu entendimento de senso comum é elevado, aproximadamente $56 \%$ do total; e os trabalhos que buscam discutir o conceito motivação de forma fundamentada mostram uma limitação dos referenciais utilizados, evidenciando alguns autores, os quais apresentam fortes vínculos acadêmicos.

PALAVRAS-CHAVE: Motivação. Análise de Conteúdo. ENPEC. 


\section{INTRODUÇÃO}

Este trabalho traz resultados de uma investigação que objetivou compreender como o conceito motivação tem sido inserido nos artigos científicos na área de pesquisa em Ensino de Ciências. Assim, realizamos um levantamento nos anais do Encontro Nacional de Pesquisa em Educação em Ciências (ENPEC), no período de 2005 a 2015, totalizando seis edições.

O ENPEC, é um evento bienal que reúne trabalhos da área de Ensino de Ciências de âmbito nacional desde 1997 (no ano de 2017 chega a sua décima primeira edição). Sendo um evento importante, considerado o maior encontro da área no país, reúne trabalhos de diversos grupos de pesquisa, o que permite uma verificação panorâmica, identificando o direcionamento das pesquisas nos principais grupos da área.

O termo motivação tem sua origem na psicologia e, segundo Todorov e Moreira (2005) é utilizado por diferentes autores em diferentes contextos, assumindo, portanto, uma diversidade de significados. Assim, no presente trabalho, não se discutiram problemas epistemológicos da conceituação. Buscouse apenas desenvolver algumas considerações sobre o conceito motivação, com foco no ambiente educacional, a fim de fundamentar nossas análises e reflexões.

De acordo com Tardif (2014) para que o aluno aprenda é necessário que de alguma forma seja inserido no processo de aprendizado, mas para que isso ocorra o aluno tem que estar motivado. A situação envolve um problema que as pesquisas denominam de motivação do aluno, na qual está envolvido um conjunto mediações complexas da interação humana.

Nesse sentido, o professor precisa mobilizar um conjunto de habilidades para orientar suas ações pedagógicas, sendo que uma dessas habilidades relaciona-se à motivação do aluno, com o objetivo de envolvê-lo no aprendizado. Porém, para que essa ação se efetive é necessária sedução, persuasão, autoridade, retórica, recompensa e punição (TARDIF, 2014). Assim, diante dessa complexa tarefa, buscamos referencias teóricos que nos auxiliassem a compreender como o conceito motivação está relacionado com processo de aprendizagem.

Com a finalidade de aprofundar como termo motivação associa-se com o processo de aprendizado, utilizamos os conceitos contemporâneos de ensino e aprendizagem de Illeris (2013), segundo os quais o aprendizado desenvolve-se por dois processos bem distintos: um processo interno para elaborar e aquisição do aprendizado; e outro processo externo de interação com o ambiente. Assim, para que haja aprendizagem os dois processos devem estar envolvidos.

O conceito de aprendizagem defendido por Illeris (2013) é basicamente de natureza construtivista, "pressupondo que o próprio aprendiz constrói ou interpreta suas estruturas mentais ativamente" (SETTI; GRANDO, 2015, p. 4) e, de acordo com Souza (2013) está fundamentado em três princípios: "a funcionalidade, relacionada ao conteúdo da aprendizagem e a compreensão do indivíduo, a sensibilidade, que acentua a importância do incentivo (motivação, emoção e vontade) e a integração, que tem como foco a interação com o outro e com o ambiente" (s/p). 
Figura 1 - As três dimensões da aprendizagem e do desenvolvimento de competências

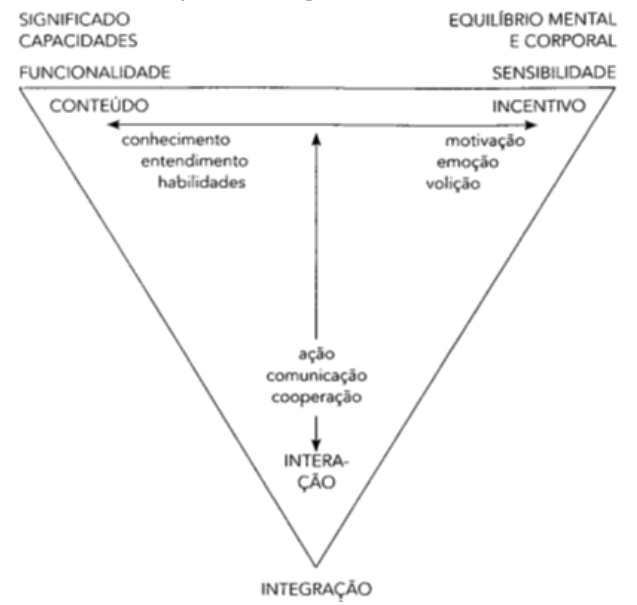

Fonte: Illeris (2013, p. 19).

A Figura 1, apresentada anteriormente, representa as três dimensões da aprendizagem e do desenvolvimento de competências, propostas por Illeris (2013): conteúdo, incentivo e interação. De acordo com autor, o conteúdo e o incentivo dependem crucialmente do processo de interação entre o indivíduo e o ambiente social e cultural, já o valor e a durabilidade da aprendizagem resultante estão intimamente conectados com a dimensão do incentivo (motivação).

A dimensão do conteúdo diz respeito àquilo que é aprendido. O indivíduo busca na dimensão conteúdo construir significado, compreensão e capacidade para lidar com desafios. Na dimensão do incentivo, é proporcionada a energia mental para o processo de aprendizado, no qual sentidos como emoção, motivação e volição (processo cognitivo pelo qual um indivíduo se decide a praticar uma ação em particular) direcionam esse processo. Já a dimensão da interação contempla a participação do estudante em atividades de cooperação e comunicação, auxiliando na construção da sociabilidade do indivíduo (ILLERIS, 2013).

Portanto, corroborando com Tardif (2014), as três dimensões da aprendizagem, apresentadas na figura 1, contemplam o processo de interação, o qual envolve a comunicação e a cooperação, que podem ser realizados por um mediador, mobilizando suas ações a fim de motivar o aluno à construção do conhecimento.

Segundo os autores Boruchovitch e Bzuneck (2009), os quais desenvolveram trabalhos na psicologia contemporânea em torno da motivação do aluno no ambiente escolar, os estudos da psicologia e das teorias contemporâneas de aprendizagem contribuem no entendimento das ações do professor no processo de interação citado por Illeris (2013), no qual o professor assume o papel de mediador do processo de aprendizagem. Assim, entender os aspectos motivacionais que influenciam a aprendizagem do aluno pode auxiliar o trabalho do professor (BORUCHOVITCH; BZUNECK, 2004). favorecer um ambiente social controlador ou promotor de autonomia. Os professores podem e devem explorar a poderosa força motivacional advinda da motivação. (BORUCHOVITCH; BZUNECK, 2004, p. 54). 
Na sala de aula podemos encontrar muitos alunos sem interesse. Essa falta de interesse pode estar relacionada à inúmeros fatores, como exemplo à obrigatoriedade da escolarização até à idade escolar prevista por lei. Diante desse cenário, o docente deve convencer o seu cliente dos benefícios de sua ação e constantemente precisa motivar e convencer que a escola é importante para o desenvolvimento dos estudantes. Portanto, compreender essa interação entre professor e aluno é fundamental para a participação do aluno no processo de ensino e aprendizagem (TARDIF; LESSARD, 2008).

A complexidade do conceito motivação vai além do uso cotidiano do termo, a motivação é importante no processo de aprendizado, ter uma dimensão dos principais grupos de pesquisas poderá evidenciar tendência, contribuições, lacunas e limitações. O levantamento poderá auxiliar professores e pesquisadores demostrando temas e problemas para novas investigações. No desenrolar desse trabalho pretende-se estabelecer relações com os dados obtidos a partir do levantamento realizado nos anais do ENPEC, buscando caracterizar algumas tendências e movimentos das pesquisas no Ensino de Ciências.

\section{METODOLOGIA}

Como metodologia de coleta e análise de dados da pesquisa utilizamos a análise textual, com ênfase na Análise de Conteúdo. O procedimento de análise textual pode ser desenvolvido com textos existentes ou entrevistas que podem ser criadas, possibilitando a apresentação de informações de forma qualitativa a partir dos dados organizados. A metodologia não tem o objetivo de testar ou rejeitar hipóteses. A intenção é reunir dados em torno de um determinado tema para analisar e compreender ou construir conhecimento em torno do tema (MORAES e GALIAZZI, 2007).

O ciclo para desenvolvimento do trabalho inicia-se com o corpus. Todo material que fez parte do acervo é analisado para criação das primeiras unidades. Na próxima etapa da análise, desenvolvem-se as unidades de significação criando categorias, sendo que o sistema de categorias é válido quando aplicado com precisão ao conjunto de informações.

O sistema de categorias possibilita uma multiplicidade das informações, podendo emergir de forma implícita ou explicita. O mesmo conjunto de significantes pode ter um conjunto de significados diferentes, as interpretações dependerão dos referencias teóricos adotados e da visão de mundo de cada pesquisador.

A categorização é um processo de comparação constante entre as unidades definidas no momento inicial da análise, levando a agrupamentos de elementos semelhantes. [...] Também implica nomear e definir as categorias, cada vez com maior precisão, na medida em que vão sendo construídas. [...] Nesse processo, as categorias vão sendo aperfeiçoadas e delimitadas cada vez com maior rigor e precisão. (MORAES; GALIAZZI, 2007, p. 22).

Com a análise é possível, por exemplo, identificar estereótipos criados em um território ou tendências de pesquisa de determinada época. As análises realizadas permitem compreender as dimensões gerais de certas produções acadêmicas, interpretação de fenômenos (BARDIN, 2004). 
De acordo com Bardin (2004), a fase interpretativa da investigação, ocorre na categorização, que é um procedimento de agrupar as inúmeras informações, que se apoiam nos avanços das compreensões e possibilitam novas reflexões ou indícios de tendências de pesquisa.

Nesse contexto, o presente trabalho tem como objetivo, a partir do uso da análise textual, levantar dados que possam nos indicar tendências e movimentos característicos dos estudos acerca da motivação no Ensino de Ciências.

A partir de um breve levantamento histórico acerca do conceito motivação apresentado por Birney e Teevan (1962, citado por TODOROV e MOREIRA, 2005), nota-se que o interesse contemporâneo da motivação humana se origina em três fontes: na psicoterapia, na psicometria e nas teorias da aprendizagem; considerando a fonte de diversidade, Todorov e Moreira (2005) trazem algumas definições encontradas na literatura, as quais serão apresentadas no quadro a seguir:

Quadro 1 - Definições do conceito de motivação

\begin{tabular}{c|c} 
Autor & \multicolumn{1}{c}{ Definição } \\
\hline Bzuneck, 2004, p. 9 & $\begin{array}{c}\text { A motivação tem sido entendida ora como um fator } \\
\text { psicológico, ou conjunto de fatores, ora como um } \\
\text { processo. Existe um consenso generalizado entre os } \\
\text { autores quanto à dinâmica desses fatores psicológicos } \\
\text { ou do processo, em qualquer atividade humana. Eles } \\
\text { levam a uma escolha, instigam, fazem iniciar um } \\
\text { comportamento direcionado a um objetivo. }\end{array}$ \\
\hline Penna, 2001, p. 19 & $\begin{array}{c}\text { Em abordagem operacional, (motivação) é o conjunto } \\
\text { de relações entre as operações de estimulação ou } \\
\text { privação e as modificações observadas no } \\
\text { comportamento que se processa após as citadas } \\
\text { operações. }\end{array}$ \\
\hline Rogers, Ludington e Graham, p. 2 & $\begin{array}{c}\text { Sempre que sentimos um desejo ou necessidade de } \\
\text { algo, estamos em um estado de motivação. Motivação } \\
\text { é um sentimento interno é um impulso que alguém } \\
\text { tem de fazer alguma coisa. }\end{array}$ \\
\hline Cofer, 1972, p. 2 & $\begin{array}{c}\text { A psicologia tende a limitar a palavra aos fatores } \\
\text { envolvidos em processos de energia, e a incluir outros } \\
\text { fatores na determinação do comportamento. }\end{array}$ \\
\hline Mudanças na significância de estímulos são a \\
preocupação básica do estudo da motivação.
\end{tabular}

Fonte: Adaptado de Todorov e Moreira (2005)

A partir das definições para o conceito motivação apresentadas no quadro anterior é possível evidenciar que esse conceito é abordado de diversas maneiras por pesquisadores das diferentes áreas do conhecimento. Especificamente no Ensino de Ciência, existe também essa diversidade de conceituações acerca do termo motivação? Os pesquisadores ou grupos de pesquisas preocupados com a temática no Brasil assumem os mesmos referenciais teóricos? Há um crescimento do número de trabalhos acerca da temática no maior encontro científico da área no país, nos últimos dez anos? 
Buscaremos, portanto, nos próximos tópicos do trabalho responder os questionamentos elencados anteriormente a partir da análise dos dados, cuja metodologia de coleta será detalhada a seguir.

\section{COLETA DE DADOS E PRIMEIRA ANÁLISE}

Realizamos buscas nos anais do Encontro Nacional de Pesquisas em Educação em Ciências (ENPEC) disponíveis na internet de forma gratuita, utilizando como disparador inicial de busca a palavra motivação. Os artigos selecionados deveriam apresentar o termo de pesquisa no título do artigo. Todavia, a seleção não se esgotou nesse processo, não encontrado no título, realizou-se uma nova busca, procurando o termo nas palavras-chave ou dentre o resumo, e quando necessário realizamos a busca da palavra no artigo.

O acesso aos anais anteriores a 2005 apresentou certa dificuldade para as pesquisas dos artigos e consultas das atas dos eventos. Observamos que todas as páginas de acesso anteriores não possuem ferramentas de busca na forma de hiperlink na página de divulgação do evento e, por esse motivo, selecionamos apenas os eventos posteriores a 2005, totalizando 06 edições.

No levantamento inicial foram encontrados 93 artigos referentes à temática da pesquisa, cujo termo pesquisado foi encontrado no título (n.1), palavras-chave (n.2) ou dentre os resumos (n.3), em um universo de 5698 artigos, conforme detalhamento apresentado no quadro 2 a seguir:

Quadro 2 - Trabalhos constituintes do corpus da pesquisa

\begin{tabular}{c|c} 
ENPEC & Trabalhos encontrados com a palavra motivação \\
\cline { 2 - 2 } & $\begin{array}{c}\text { 01.Ensino-aprendizagem da geometria analítica: uma proposta utilizando o } \\
\text { GPS como recurso didático e fator de motivação da aprendizagem. Bezerra, } \\
\text { N. J. F.; Scartazzini, L. S. (n.1) }\end{array}$
\end{tabular}

02. Unidade de aprendizagem: uma alternativa para professores e alunos conviverem melhor. Albuquerque, Fernanda Medeiros; Basso, S. B. N. F.; Azambuja, R.; Basso, N. R. S. (n.2)

03. Processos interativos em aulas de física: um estudo inicial. Monteiro, I. I. C. C.I; Gaspar, A. (n.2)

04. A mediação teórica-conceitual de um site para alunos-trabalhadores da saúde. Menezes, S.F.; Azevedo, A. C. P. (n.3)

05. Análise Da Influência Das Crenças Motivacionais De Professores De Física Do Ensino Médio. Barros, M. A. (n.1)

06. Análise, desenvolvimento e aplicação de recursos de informática no ensino de química. Merçon, F.; Souza, M. P.; Fernandez, M. M; Araújo, S.B.N.; Guimarães, P.I.C. (n.3)

07. Aprendizagem baseada em problemas no Ensino de ciências: a mudança de atitude de alunos e professores. Malheiro J. M. S.; Diniz, C.W.P (n.3)

08. Concepções dos agentes de saúde da divisão de informação, educação e comunicação do município de Niterói, sobre vermes, areias e saúde. Proença, E. C. A; Bissagio; R. C. (n.3)

09. Diferenças raciais: o que diz a biologia, o que pensam os alunos. Vieira, E. P. P; Chaves, S. N. (n.3) 


\begin{tabular}{|c|c|}
\hline ENPEC & Trabalhos encontrados com a palavra motivação \\
\hline & $\begin{array}{l}\text { 11. Ensinando e aprendendo sobre vertebrados: uma experiência de } \\
\text { docência em prática de ensino de biologia. Rechetelo, J; Luz, A. A.; Probst, A. } \\
\text { P. (n.3) }\end{array}$ \\
\hline & $\begin{array}{l}\text { 12. Formação continuada de professores de química: qual modelo, qual } \\
\text { formação? Bruno F. S.; Luana N. S. (n.3) }\end{array}$ \\
\hline & $\begin{array}{l}\text { 13. Metodologia de projetos e ambientes não formais de aprendizagem: } \\
\text { indício de eficácia no processo do ensino de biologia. Oliveira, C. L.; Moura, } \\
\text { D. E. (n.3) }\end{array}$ \\
\hline & $\begin{array}{l}\text { 14. Modelagem matemática e resolução de problemas nas ciências com } \\
\text { equações diferenciais. Laudares, J. B. (n.3) }\end{array}$ \\
\hline & $\begin{array}{l}\text { 15. Temas conectores: uma alternativa ao modelo clássico de ensino de } \\
\text { física. Alencar, J.R.S; Oliveira, S. H. B. (n.3) }\end{array}$ \\
\hline & $\begin{array}{c}\text { 16. Unidade de aprendizagem: uma alternativa para professores e alunos } \\
\text { conviverem melhor. Albuquerque, F. M; Ferraro, S.B.C.S; Azambuja, R; } \\
\text { Basso, N. R. S. (n.2) }\end{array}$ \\
\hline & $\begin{array}{l}\text { 17. Espaços não-formais: motivação para a aprendizagem do ensino de física } \\
\text { promovendo a educação científica. Castro, J. F. A.; Fernandes, D.C.; } \\
\text { Marques, R. N.; Las, W. C.; Oliveira L. A. A. (n.1) }\end{array}$ \\
\hline & $\begin{array}{l}\text { 18. Experimento cativante: Critérios para a elaboração tendo como base a } \\
\text { teoria da motivação. Laburú, C. E. (n.1) }\end{array}$ \\
\hline & $\begin{array}{l}\text { 19. Interesse de estudantes sobre temas de biologia. Mendes, I; Borges, } \mathrm{O} \text {. } \\
\qquad(\mathrm{n} .02)\end{array}$ \\
\hline & $\begin{array}{l}\text { 20. Processos interativos em aulas de física: um estudo inicial. Monteiro, I. } \\
\text { C. C; Gaspar, A. (n.2) }\end{array}$ \\
\hline \multirow{12}{*}{2007} & $\begin{array}{l}\text { 21.A motivação do aluno para aprender conteúdos de física na sua relação } \\
\text { com o uso de projetos escolares experimentais. Espindola, A. C., A.; Dias, A. } \\
\text { C. G.; Barlette, V. E. (n.2) }\end{array}$ \\
\hline & $\begin{array}{l}\text { 22.Matemática como estruturante e física como motivação: uma análise de } \\
\text { concepções sobre as relações entre matemática e física. Karam, R. A. S. (n.2) }\end{array}$ \\
\hline & $\begin{array}{l}\text { 23. Motivação ao magistério e identidade de professores que ensinam } \\
\text { ciências em um país em reconstrução: angola/cabinda. Buza, J. L. C.; } \\
\text { Gonçalves, T. V. O. (n.2) }\end{array}$ \\
\hline & $\begin{array}{l}\text { 24.Motivação e interação social: o discurso do professor em uma atividade } \\
\text { de demonstração. Monteiro, M. A. A.; Monteiro, I. C. C.; Villani, A.; Gaspar } \\
\text { A. (n.2) }\end{array}$ \\
\hline & $\begin{array}{l}\text { 25.Relações entre motivação, valor e perfil conceitual: um exemplo. Dalri, } \\
\text { J.; Mattos, Rodrigues, C. (n.2) }\end{array}$ \\
\hline & $\begin{array}{l}\text { 26.Um estudo sobre a emoção e a motivação em aulas de física. Monteiro, } \\
\text { Isabel, C. C.; Gaspar, A.; Monteiro, M. A. A.; Villani, A. (n.2) }\end{array}$ \\
\hline & $\begin{array}{l}\text { 27. Análise da elaboração e aplicação de uma sequência didática sobre o } \\
\text { aquecimento global. Vilela, C. X.; Guedes, M. G. M; Amaral, E. M. R; } \\
\text { Barbosa, R. M. N. (n.3) }\end{array}$ \\
\hline & $\begin{array}{l}\text { 28. Análise do contexto de criação de aparatos interativos em museus de } \\
\text { ciência. Falcão, B. (n.3) }\end{array}$ \\
\hline & $\begin{array}{l}\text { 29. Aprender ciências em grupo: o que os alunos pensam? Guedes, M. G. M; } \\
\text { Barbosa, R. M. N; Jófili, Z. M. S. (n.3) }\end{array}$ \\
\hline & $\begin{array}{l}\text { 30. Discussão conceitual em espaços assimétricos de formação. Belisario, C. } \\
\text { S.; Echeverría, A. R. (n.3) }\end{array}$ \\
\hline & $\begin{array}{l}\text { 31. Educação em centros de ciências: visitas escolares ao Observatório } \\
\text { astronômico do CCDC/USP. Junior, P. D. C.; Aroca, S. C.; Silva, C. C. (n.3) }\end{array}$ \\
\hline & $\begin{array}{l}\text { 32. Projeto integrado sobre aquecimento global e mudança de postura dos } \\
\text { apreendestes. Duso, L.; Borges, R. M. R. (n.3) }\end{array}$ \\
\hline
\end{tabular}




\begin{tabular}{|c|c|}
\hline ENPEC & Trabalhos encontrados com a palavra motivação \\
\hline & $\begin{array}{l}\text { 33. O texto de divulgação científica como recurso para o ensino de ciências } \\
\text { na educação básica: um levantamento das produções nos ENPEC. Puiati, L. } \\
\qquad \text { L; Borowsky, H. G.; Terrazzan, E. A. (n.3) }\end{array}$ \\
\hline & $\begin{array}{l}\text { 34. Uma análise das diferenças de gênero no discurso escolar Junior, P. R. } \\
\text { M.; Ostermann, F.; Rezende, F.; Cavalcanti, C. J. H. (n.3) }\end{array}$ \\
\hline & $\begin{array}{l}\text { 35. Humano, seus direitos e o discurso biológico moderno. Vieira, E. P. P.; } \\
\text { Chaves, S. N. (n.3) }\end{array}$ \\
\hline \multirow{7}{*}{2009} & $\begin{array}{l}\text { 36. Aprendizagem cooperativa no ensino de química: uma proposta de } \\
\text { abordagem em sala de aula. Silva, A. J.; Gauche, R. (n.3) }\end{array}$ \\
\hline & $\begin{array}{l}\text { 37. Atividades de campo no ensino de ciências e na educação ambiental: } \\
\text { aspectos da prática educativa de um grupo de professores. Viveiro L. A.; } \\
\text { Diniz, R. E. S. (n.3) }\end{array}$ \\
\hline & $\begin{array}{l}\text { 38. Autoria no ensino superior de química: investigando uma atividade } \\
\text { pautada no uso de textos de divulgação científica. Ferreira, L. R. A.; Queiroz, } \\
\text { S. L. (n.3) }\end{array}$ \\
\hline & $\begin{array}{l}\text { 39. As olimpíadas científicas: motivação para o estudo da química? Quadros, } \\
\text { A. L.; Silva, D. C.; Andrade, F. P.; Silva, G. F.; Aleme, H. G.; Oliveira, S. R.; } \\
\text { Fátima, A. (n.1) }\end{array}$ \\
\hline & $\begin{array}{l}\text { 40. Novos sentidos para velhas questões: limites e possibilidades de uma } \\
\text { proposta de ensino desenvolvida durante o estágio supervisionado em } \\
\text { ciências. Paula, G. S. (n.3) }\end{array}$ \\
\hline & $\begin{array}{l}\text { 41.Reflexão sobre a interdependência dos processos de ensino- } \\
\text { aprendizagem e avaliação da aprendizagem de alunos ingressantes de curso } \\
\text { de física. Costa, S. S. C.; Tarragó M. E. P; Santos L. (n.3) }\end{array}$ \\
\hline & $\begin{array}{l}\text { 42. Utilização de recursos multimidiáticos e web } 2.0 \text { para o ensino de } \\
\text { astronomia uma experiência com professores de física. Siqueira B. A.; Rojas } \\
\text { G. A.; Oliveria A. J. A. (n.3) }\end{array}$ \\
\hline \multirow{10}{*}{2011} & $\begin{array}{l}\text { 43.As motivações de professores de ciências para a formação contínua a } \\
\text { distância. Garcia P.S; Bizzo N. (n.1) }\end{array}$ \\
\hline & $\begin{array}{l}\text { 44.Licenciatura em Química: carência de professores, condições de trabalho } \\
\text { e motivação pela carreira docente. Sá C. S.; Santos,W.L.P. (n.1) }\end{array}$ \\
\hline & $\begin{array}{l}\text { 45.Motivação para estudar química: configurações subjetivas de uma } \\
\text { estudante do segundo ano do ensino médio. Pessoa, W.R; Alves J.M. (n.1) }\end{array}$ \\
\hline & $\begin{array}{l}\text { 46.Sentidos subjetivos relacionados com a motivação dos estudantes do } \\
\text { Clube de Ciências da Ilha de Cotijuba. Alves J.M; Pessoa W.R; Rodrigues } \\
\text { A.M.S; Santos,J.K.R (n.1) }\end{array}$ \\
\hline & $\begin{array}{l}\text { 47. A Literatura de Cordel como veículo de popularização da ciência: uma } \\
\text { intervenção no ensino de Física. Lima, J. M.; Sousa J. M.; Germano M. G. } \\
\text { (n.3) }\end{array}$ \\
\hline & $\begin{array}{l}\text { 48. Ações formativas em Educação Ambiental: análise de um trabalho na } \\
\text { rede municipal de ensino. Bonzanini T. K.; Ribeiro I. C.; Rodrigues, C. S. (n.3) }\end{array}$ \\
\hline & $\begin{array}{l}\text { 49. Brinca Ciência: Um Ensaio Lúdico Educativo Sobre Ciência \& Tecnologia } \\
\text { na Escola Pública do Município de Santo André. Neto A. F. F.; Gomes A. A.; } \\
\text { Augusta, B. G.; Mamede F. R. S.; Rodrigues, J.; Friolani, P. (n.3) }\end{array}$ \\
\hline & $\begin{array}{l}\text { 50. Inovação curricular de Física Moderna: motivações, dificuldades e } \\
\text { mudanças na prática docente. Souza A. P. G.; Lawall, I. T. (n.1) }\end{array}$ \\
\hline & $\begin{array}{l}\text { 51. Limiares de tolerância à sujeira, destilação e o ensino das ciências: uma } \\
\text { perspectiva histórico-cultural. Almeida, R. O. (n.3) }\end{array}$ \\
\hline & $\begin{array}{l}\text { 52. O Uso de jogos como Ferramenta de apoio no processo de Ensino- } \\
\text { aprendizagem da Matemática. Pessanha, P. R.; Peres, A. M. F.; D’Alverga, M; } \\
\text { Souza L. F.; Santos R. (n.3) }\end{array}$ \\
\hline
\end{tabular}




\begin{tabular}{|c|c|}
\hline ENPEC & Trabalhos encontrados com a palavra motivação \\
\hline & $\begin{array}{l}\text { 53. Os sentidos atribuídos ao ensino por investigação por professores não } \\
\text { especialistas em formação inicial. Garcia J. F. M.; Trazzi, P. S. S. (n.3) }\end{array}$ \\
\hline & $\begin{array}{l}\text { 54. Possibilidades lúdicas em um espaço de educação não formal. Faria, R. } \\
\text { L.; Shuvartz, M. (n.3) }\end{array}$ \\
\hline & $\begin{array}{l}\text { 55. Proposta de sequência didática para o ensino de astronomia no } \\
\text { fundamental: Conhecendo a lua. Santos, J. H. M.; Pereira F. N. V.; Penido, M } \\
\text { C. M. (n.3) }\end{array}$ \\
\hline & $\begin{array}{l}\text { 56. Realidade aumentada no ensino de ciências: tecnologia auxiliando a } \\
\text { visualização da informação. Rolim A. L. S; Rodrigues R. L.; Oliveira, W; Farias } \\
\text { D. S. (n.3) }\end{array}$ \\
\hline & $\begin{array}{l}\text { 57. Sentidos subjetivos da docência e motivação de professores de biologia } \\
\text { do 1o. ano do ensino médio. Alves, J. M.; Silva, D. D. R.; Martins F. F.; Pereira } \\
\text { J. S. S; Santos, M. T.; Campos, M. A. L. (n.1) }\end{array}$ \\
\hline & $\begin{array}{l}\text { 58. Uma proposta de feira de ciências para alunos do ensino médio } \\
\text { orientada pela Aprendizagem Baseada em Problemas. Salvador, D. F.; } \\
\text { Oliveira, D. B; Gustavo, L; Rolando, R; Rolando, R. F. R; Magarão J. F. L. (n.3) }\end{array}$ \\
\hline & $\begin{array}{l}\text { 59. Uma relação entre a metodologia do projeto "abc na educação cientifica } \\
\text { mão na massa" e a teoria de Jean Piaget. Souza, R. F.; Amauro N. Q.; } \\
\text { Curvelo, A. A. S. (n3) }\end{array}$ \\
\hline \multirow{13}{*}{2013} & $\begin{array}{l}\text { 60.A motivação para aprender ciências como produção subjetiva inserida na } \\
\text { cultura científica escolar; Alves J. M. (n.1) }\end{array}$ \\
\hline & $\begin{array}{l}\text { 61.Cinema como recurso didático: motivação nas aulas de ensino de } \\
\text { ciências. Santos, J. Nunes; Gebara, M. J. Fontana. (n.1) }\end{array}$ \\
\hline & $\begin{array}{l}\text { 62.Compreensão de sentidos subjetivos em aulas de campo que motivam a } \\
\text { aprendizagem de ciências. Trevisan, I; Alves J. M. (n.1) }\end{array}$ \\
\hline & $\begin{array}{l}\text { 63.Experimentos de Ciências nos Anos Iniciais do Ensino Fundamental: uma } \\
\text { ferramenta para a motivação em sala de aula. Soares, K. C.; Paula, L. M.; } \\
\text { Paula, L. M.; Silva, R. C.; Pereira, G. R. (n.1) }\end{array}$ \\
\hline & $\begin{array}{l}\text { 64.Motivação no estudo da química: sentidos subjetivos de um estudante } \\
\text { do 2o ano do ensino médio. Pessoa, W. R.; Alves J. M. (n.1) }\end{array}$ \\
\hline & $\begin{array}{l}\text { 65.Uma avaliação sobre a opinião e a motivação dos estudantes que } \\
\text { participaram de um Show de Física. Vieira, S. T.; Siman, M.; Ambrózio, R. } \\
\text { (n.1) }\end{array}$ \\
\hline & $\begin{array}{l}\text { 66.Uma estratégia de investigação multimodal para física. Goya, A.; Laburú, } \\
\text { C. E. (n.2) }\end{array}$ \\
\hline & $\begin{array}{l}\text { 67.A evolução de um projeto com o uso de recursos multimídias no ensino } \\
\text { de biologia. Rehem, H. M. F.; Cunha, H. O. C.; Grandhi, M.; Lopes, P. C. T.; } \\
\text { Nascimento, A. M. J. A. M.; Rocha, I. D. B.; Kreismann, A. C. P.; Avanzi, M. R.; } \\
\text { Gastal, M. L. (n.3) }\end{array}$ \\
\hline & $\begin{array}{l}\text { 68.Efeitos da discussão de conceitos por professores num espaço coletivo. } \\
\text { Mariano, I. A.; Rossi, A. V. (n.2) }\end{array}$ \\
\hline & 69.Subjetividade e pedagogia de projetos. Casanova, M. P.; Alves, J. M. (n.2) \\
\hline & $\begin{array}{l}\text { 70.Aulas de campo: uma estratégia de ensino necessária? Hencklein, A. H. } \\
\text { (n.2) }\end{array}$ \\
\hline & $\begin{array}{l}\text { 71. O ensino de conceitos sobre ritmos biológicos no ensino médio a partir } \\
\text { do conteúdo “Características gerais dos seres vivos. Filho P. L. S.; Souza J. P. } \\
\text { P.; I. C. S. Guimarães; Costa I. A. S.; Azevedo C. V. M.; Zuza, H. O. B. B. (n.3) }\end{array}$ \\
\hline & $\begin{array}{l}\text { 72. A física também é ciência: as experiências do estágio e a percepção } \\
\text { sobre o ensino de ciências nos anos iniciais. Santos J. K. R.; Cajueiro, D. D. S; } \\
\text { Santos, V. B; Gemaque, R. F. L; Rocha P. J. C. (n.3) }\end{array}$ \\
\hline
\end{tabular}




\begin{tabular}{|c|c|}
\hline ENPEC & Trabalhos encontrados com a palavra motivação \\
\hline & $\begin{array}{l}\text { 73. A vivência no museu de Ciências sob a perspectiva do Modelo } \\
\text { Contextual de Aprendizagem: um estudo de caso. Souza, V. M.; Silva, A. M. } \\
\text { M.; Ramos, M. G. (n.3) }\end{array}$ \\
\hline & $\begin{array}{l}\text { 74. Agrotóxicos - toxidade versus custos: uma experiência de formação de } \\
\text { professores com as questões sociocientíficas no ensino de ciências. Lopes, } \\
\text { N. C.; Carvalho, W. L. P.; Faria, P. C. (n.3) }\end{array}$ \\
\hline & $\begin{array}{l}\text { 75. Aplicação e teste de uma sequência didática sobre sistema sanguíneo } \\
\text { ABO no ensino médio de biologia. Pinheiro, S. A.; Costa I. A. S.; Silva M. F. } \\
\text { (n.3) }\end{array}$ \\
\hline & $\begin{array}{l}\text { 76. H'Química - O uso dos quadrinhos para o Ensino de Radioatividade. } \\
\text { Cruz, T. M. G. S; Mesquita, N. A. S; Soares, M. H. F.B. (n.3) }\end{array}$ \\
\hline & $\begin{array}{l}\text { 77. Identificação de conceitos astronômicos em livros paradidáticos na } \\
\text { formação de professores de Ciências. Sanzovo, D. T; Laburú, C. E. (n.3) }\end{array}$ \\
\hline & $\begin{array}{l}\text { 78. Modelos atômicos em livros didáticos de química do PNLEM 2012: uma } \\
\text { análise qualitativa à luz da história e filosofia da ciência. Moura, C. B.; } \\
\text { Guerra, A. (n.3) }\end{array}$ \\
\hline & $\begin{array}{l}\text { 79. O papel motivador e problematizador da observação da cortiça } \\
\text { enquanto episódio na História da Biologia: uma análise das interações } \\
\text { discursivas. Klepka, V.; Corazza, M. J. (n.1) }\end{array}$ \\
\hline \multirow{12}{*}{2015} & $\begin{array}{l}\text { 80. A Licenciatura por acaso: motivações para o ingresso em um curso de } \\
\text { Licenciatura e Bacharelado em Química. Miranda, C. L.; Lisbôa, J. C. F.; } \\
\text { Rezende, D. B. (n.1) }\end{array}$ \\
\hline & $\begin{array}{l}\text { 81. A Motivação e o Interesse de estudantes em aulas de Ciências na } \\
\text { perspectiva do Ensino por Investigação. Oliveira, S. G.T. Q; Neves, M. L. R. C. } \\
\text { (n.1) }\end{array}$ \\
\hline & $\begin{array}{l}\text { 82. Análise de aspectos da Natureza da Ciência (NdC) e motivacionais em } \\
\text { estudantes do Ensino Médio mediada por sequência didática centrada na } \\
\text { replicação de experimentos históricos darwinianos. Nogueira, L. V.; Freitas, } \\
\text { K. C. (n.1) }\end{array}$ \\
\hline & $\begin{array}{l}\text { 83. Análise de recursos e situações de ensino: a motivação para aprender. } \\
\text { Moura, P.; Bonzanini, K. T. (n.1) }\end{array}$ \\
\hline & $\begin{array}{l}\text { 84. Motivações de Professores da Área de Ciências da Natureza e } \\
\text { Matemática para Cursar uma Especialização em Ensino de Ciências. Michels, } \\
\text { J.; Farias, M. E. (n.1) }\end{array}$ \\
\hline & $\begin{array}{l}\text { 85. Motivação para aprender e ensinar química: configurações subjetivas de } \\
\text { um professor do ensino médio. Pessoa, W. R.; Alves, J. M. (n.1) }\end{array}$ \\
\hline & $\begin{array}{l}\text { 86. Por que professores de Ciências visitam museus? Um estudo de caso } \\
\text { sobre a percepção de professores de Campinas-SP e Duque de Caxias-RJ. } \\
\text { Pinto, L. T.; Rossi, A. V. (n.2) }\end{array}$ \\
\hline & $\begin{array}{l}\text { 87.Traços motivacionais como orientação para a prática docente. } \\
\text { Fernandes, S. J.; Rodrigues, E. V.; Camiletti, G. (n.1) }\end{array}$ \\
\hline & $\begin{array}{l}\text { 88. Uma Investigação sobre a Aprendizagem Baseada em Problemas nas } \\
\text { Ciências da Natureza: percepções de um grupo de estudantes do Ensino } \\
\text { Médio. Lima, D. B.; Valentim, L. M. (n.2) }\end{array}$ \\
\hline & $\begin{array}{l}\text { 89. Pedagogia de projetos, teatro e motivação nas aulas de Ciências. } \\
\text { Casanova, M. P.; Alves J. A. (n.1) }\end{array}$ \\
\hline & $\begin{array}{l}\text { 90. Articulando Química, questões raciais e de gênero numa Oficina sobre } \\
\text { Diversidade desenvolvida no âmbito do PIBID: análise da contribuição dos } \\
\text { recursos didáticos alternativos. Santos, R. G.; Siemsen, G. H.; Silva, C. S. (n.3) }\end{array}$ \\
\hline & $\begin{array}{l}\text { 92. As trilhas ecológicas e o ensino de ciências: análises dos últimos anais } \\
\text { dos encontros de Ensino de Ciências, Biologia e Educação Ambiental no } \\
\text { Brasil. Santos, F. C.; Silva, F. A. R. (n.3) }\end{array}$ \\
\hline
\end{tabular}




\section{ENPEC Trabalhos encontrados com a palavra motivação \\ 91. Educação Ambiental: perfil e perspectivas motivadoras de um grupo de sujeitos na constituição de espaços educadores sustentáveis. Gil, R. L; Behling, G. M; Carlan, F. A. (n.3) \\ 93. O papel da História da Ciência no Ensino de Química: ideias iniciais de futuros professores. Stanzani, L. E.; Guarnieri, P. V.; Broietti, F. C. D.; Bastos, F. (n.3)}

Legenda: (n.1) - Artigo que apresenta a palavra motivação no Título; (n.2) - Artigo que apresenta a palavra motivação na palavras-chave; (n.3) - Artigo que apresenta a palavra motivação no resumo.

Fonte: Autoria própria (2017).

A organização do Quadro 2 possibilitou a separação dos artigos publicados ao longo de 10 anos do evento, constituindo o nosso corpus de análise, com 93 artigos. 0 total de trabalhos listados que apresentam o termo motivação corresponde a aproximadamente $1,6 \%$ do total de publicações correspondentes às seis edições do ENPEC pesquisadas, evidenciando a pouca representatividade das pesquisas referentes ao tema na última década.

A pouca representatividade talvez tenha relação com limitações educacionais acerca do conceito motivação, conforme apresentado por Boruchovitch e Bzuneck (2004). Segundo os autores, essas limitações podem estar conectadas à ângulos diversos do conceito motivação que foram criados ao longo da construção histórica do tema e à limitada quantidade de referências teóricos sobre motivação no contexto educacional.

Os dados apresentados no Quadro 2 não mostram uma variação expressiva do número de trabalhos que contemplam o termo motivação nas últimas seis edições do ENPEC. Nesse sentido, realizamos um segundo movimento de organização dos dados, a fim de aprofundar essa constatação.

Na Tabela 1 - a seguir - apresentamos os dados quantitativos referentes à quantidade de artigos submetidos e artigos aceitos nas edições pesquisadas essas informações encontram-se disponíveis no site do evento. Também adicionamos o número total de trabalhos que constituem o corpus da presente investigação, separando-os por biênio.

Tabela 1 - Informações quantitativas sobre a coleta dados no ENPEC

\begin{tabular}{ccccc}
\hline Ano & $\begin{array}{c}\text { Submetidos no } \\
\text { evento }\end{array}$ & Aceitos no evento & $\begin{array}{c}\text { Total de artigos } \\
\text { selecionados } \\
\text { sobre motivação }\end{array}$ & $\%$ \\
\hline 2005 & 834 & 739 & 20 & 2,7 \\
\hline 2007 & 958 & 669 & 15 & 2,2 \\
\hline 2009 & 1140 & 723 & 7 & 1,0 \\
\hline 2011 & 1695 & 1235 & 17 & 1,4 \\
\hline 2013 & 1526 & 1060 & 20 & 1,9 \\
\hline 2015 & 1768 & 1272 & 14 & 1,1 \\
\hline Total & 7921 & 5698 & 93 & 1,6 \\
\hline
\end{tabular}

Fonte: Autoria própria (2017) 
A partir da análise dos dados apresentados na Tabela 1 evidencia-se que, embora a quantidade de trabalhos relativos à temática motivação não apresenta alterações consideráveis ao longo das edições. Porém, visto que o número de artigos submetidos e aceitos apresenta um crescimento ao longo dos anos, logo, proporcionalmente, há uma queda importante, uma vez que o evento passa a aceitar cerca de $40 \%$ mais trabalhos se compararmos as edições de 2005 e 2015 , especificamente.

Em um terceiro movimento analítico, apresentado na Tabela 02, os 93 trabalhos foram separados de acordo com a busca realizada, considerando se o termo motivação foi encontrado no título, nas palavras-chave ou no resumo dos trabalhos.

Tabela 2 - Elemento do texto no qual o termo motivação foi encontrado

\begin{tabular}{ccccc} 
Ano & $\begin{array}{c}\text { Total de } \\
\text { artigos }\end{array}$ & Título & Palavras-chave & Resumo \\
\hline 2005 & 20 & 4 & 5 & 11 \\
\hline 2007 & 15 & 6 & 0 & 9 \\
\hline 2009 & 7 & 1 & 0 & 6 \\
\hline 2011 & 17 & 5 & 1 & 11 \\
\hline 2013 & 20 & 7 & 4 & 9 \\
\hline 2015 & 14 & 9 & 1 & 4 \\
\hline Total & 93 & 32 & 11 & 50 \\
\hline
\end{tabular}

Fonte: Autoria própria (2017)

A quantidade elevada de artigos em que o termo motivação aparece apenas no resumo, aproximadamente $54 \%$, induziu um aprofundamento da análise, iniciando outro movimento de verificação dos dados. Procuramos nos 93 trabalhos informações acerca dos referenciais teóricos utilizados nas discussões do conceito motivação, porém, percebemos que nem todos os autores buscaram fundamentar o conceito, ou seja, o termo era utilizado com um sentido de senso comum.

Na sequência, realizamos a leitura de todos os trabalhos que traziam citações de referenciais diretos a fim de definir o conceito motivação. Esse aprofundamento analítico possibilitou um novo conjunto de informações.

Organizamos os trabalhos que utilizam referencial teórico a partir de um código de identificação. O código inicia-se com os dois primeiros números referentes ao ano de ocorrência do evento e, em seguida, adicionamos o número de identificação do artigo conforme o Quadro 2 - exemplo: 0 trabalho 22, publicado nos anais do ENPEC de 2007, foi identificado pelo código 07.22. O Quadro 4 traz a relação desses trabalhos. 
Quadro 4 - Informações dos artigos com referenciais para o conceito motivação

\begin{tabular}{|c|c|c|}
\hline $\begin{array}{l}\text { Local do artigo com o } \\
\text { termo motivação }\end{array}$ & Artigos com referenciais & Total \\
\hline Título com referencial & $\begin{array}{c}05.01 ; 05.05 ; 05.18 ; 07.21 ; 07.25 ; 07.26 ; 09.39 \\
11.43 ; 11.45 ; 11.46 ; 13.60 ; 13.62 ; 13.63 ; 13.64 ; 13.6 \\
9 ; \\
15.81 ; 15.84 ; 15.85 ; 15.87 ; 15.89\end{array}$ & 20 \\
\hline $\begin{array}{l}\text { Palavras-Chave com } \\
\text { referencial direto } \\
\end{array}$ & $\begin{array}{c}05.03 ; 05.19 ; 05.20 ; 11.44 ; 13.68 ; 13.70 ; 15.86 \\
15.88 \\
\end{array}$ & 8 \\
\hline $\begin{array}{c}\text { Resumo com referencial } \\
\text { direto }\end{array}$ & 07.29; 09.37;11.54;11.57; 15.91 & 5 \\
\hline
\end{tabular}

Fonte: Autoria própria (2017)

Dos 32 artigos publicados que traziam o termo motivação no título, 20 apresentaram referenciais diretos do conceito; nas palavras-chave 8 dos 11 trabalhos apresentaram fundamentação teórica para o conceito em questão.

No trabalho 05.01 - o termo motivação é citado no título - Bezerra e Scartazzini (2005) tem como objetivo "[...] demonstrar a aplicabilidade dos conteúdos da geometria analítica e fornece ao professor mecanismos para motivar e facilitar a aprendizagem em sala de aula" (p.1). Assim, a fim de discutir o papel da motivação na aprendizagem dos conceitos, buscam fundamentar-se nas autoras Sueli Guimarães e Evely Boruchovith.

O trabalho 13.66 - o termo motivação é citado nas palavras-chave - Goya e Laburú (2017) “ [...] aprofundamento na aprendizagem por multimodos e múltiplas representações, bem como aplicar essa estratégia em acadêmicos de licenciatura em química e avaliar quanto aos conceitos newtonianos e quanto à sua motivação e estratégia de estudo para a disciplina de física 1 foi o objetivo do presente estudo. " (p.4). Para discutir a motivação e estratégias de disciplina de física, buscam fundamentar-se José Aloyseo Bzuneck.

Do total de trabalhos em que o termo motivação aparece exclusivamente no resumo, apenas cinco apresentam referencial teórico, utilizando o termo motivação como conceito, não no sentido do senso comum. Por exemplo, no trabalho 15.93, Stanzani et al. (2015) apresentam um levantamento das ideias iniciais de futuros professores de Química acerca do uso da História da Ciência no ensino. No resumo, os autores citam o termo motivação, afirmando que esse seria um dos objetivos elencados pelos licenciandos para a inserção de uma abordagem histórica, possibilitando "a motivação dos estudantes para o ensino de Química" (p.1), mas não aprofundam as discussões sobre o conceito.

O trabalho de 11.54 está entre os cinco artigos citados no Quadro 4 que trazem o termo motivação no resumo e no decorrer do trabalho utilizam uma fundamentação teórica para definir o conceito. $\mathrm{Na}$ investigação realizada o autor busca compreender como o "[...] uso de diferentes espaços e estratégias se torna um importante aliado para despertar nos alunos interesse e motivação em relação ao Ensino de Ciências." (p.4). A fim de conceituar o termo motivação, fundamentase nos autores Tatiana Seniciato e Osmar Cavassan.

Sendo assim, diante desse movimento interpretativo citado, iniciamos a leitura de todos os trabalhos que possuíam referenciais teóricos, buscamos evidenciar os autores que trabalhavam diretamente com o conceito motivação. 
Todos os referencias nacionais e internacionais citados foram considerados na análise.

Para construção do Tabela 3 - apresentada na sequência - consideramos os referenciais teóricos que foram citados mais de 5 vezes ao longo dos 10 anos de análise, com o objetivo de encontrarmos os autores mais representativos citados nos trabalhos das últimas seis edições do ENPEC.

Nos trabalhos em que o conceito motivação era definido por dois ou mais autores, esses foram individualizados e organizados na Tabela 3. Caso o mesmo autor fosse citado duas vezes utilizando obras diferentes, contabilizamos como duas citações.

Segue um exemplo encontrado no artigo 15.81, conforme quadro 4, no referencial encontramos: GUIMARÃES, S. É. R. e BORUCHOVITCH, E. (2004). O estilo motivacional do professor e a motivação intrínseca dos estudantes: uma perspectiva da Teoria da Autodeterminação. Rev. Psicologia: Reflexão e Crítica, v. 17 (2), p. 143-150. Para organizar a Tabela 3 contabilizamos uma citação para GUIMARÃES, S. É. R. e uma citação para BORUCHOVITCH, E. Caso houvesse outra citação do mesmo autor, no mesmo artigo, foram contabilizadas duas citações.

Tabela 3 - Informações dos referenciais mais citados nos artigos coletados do ENPEC

\begin{tabular}{|c|c|c|}
\hline Autor & Artigos com referenciais & $\begin{array}{l}\text { Total de } \\
\text { citações }\end{array}$ \\
\hline BZUNECK, J. A. & $\begin{array}{c}07.21 ; 11.45 ; 11.57 ; 13.60 ; 13.65 ; 13.6615 \\
81 ; 15.05 ; 15.05 ; 15.05 ; 15.20 ; 15.8115 .87 ; \\
15.87 ; 15.87 ; 15.87 ; 15.87\end{array}$ & 17 \\
\hline GUIMARÃES, S. É. R. & $\begin{array}{c}07.21 ; 11.57 ; 13.60 ; 13.65 ; 15.01 ; 15.03 ; \\
15.18 ; 15.20 ; 15.20 ; 15.26 ; 15.26 ; 15.87 ; 15 . \\
87 ; 15.88 ; 15.81 ; 15.85 ; 15.87\end{array}$ & 17 \\
\hline BORUCHOVITCH, E. & $\begin{array}{c}15.01 ; 15.03 ; 15.85 ; 11.57 ; 15.05 ; 15.20 ; 15 . \\
20 ; 15.26 ; 15.81 ; 15.87 ; 15.88\end{array}$ & 11 \\
\hline
\end{tabular}

Fonte: Autoria própria (2017).

Para um aprofundamento interpretativo acerca dos 3 autores com maior representatividade, realizamos uma pesquisa dos currículos utilizando a plataforma lattes disponível na internet de forma gratuita. Observamos que os três são graduados em psicologia apresentam uma conexão acadêmica com diversos trabalhos realizados em parceria acerca do tema motivação do aluno. Eles compartilham teses, dissertações e livros acerca do tema e, portanto, colaboraram e influenciaram os trabalhados relacionados no ensino de ciências acerca do conceito motivação do aluno no período selecionado para a análise.

\section{CONSIDERAÇÕES FINAIS}

Uma das dificuldades inerentes a esses movimentos de pesquisa, diz respeito à falta de padronização no formato das páginas de divulgação do ENPEC. A busca de informações referentes ao tema de pesquisa foi facilitada a partir da edição realizada em 2009. Nesse ano houve uma mudança na organização e forma de apresentação dos trabalhos publicados nos anais, as quais foram mantidas ao longo das edições. 
Segundo os dados levantados, percebeu-se um aumento na quantidade de trabalhos enviados ao ENPEC ao longo das edições analisadas, porém, proporcionalmente, há uma queda importante nos trabalhos relacionados à temática da investigação, cerca de $40 \%$.

Conforme as leituras transcorreram, novas organizações de dados foram emergindo e possibilitando novas visões. Por meio da análise realizada, evidenciase a limitação de referenciais utilizados nos trabalhos no Ensino de Ciências, enfatizada pela análise dos currículos dos pesquisadores, uma vez que esses possuem conexões acadêmicas, as quais podem influenciar e limitar a visão teórica e as abordagens acerca do tema.

Essa possível limitação poderia justificar a apresentação do termo sem a definição do conceito a partir do uso de referenciais diretamente citados em aproximadamente $56 \%$ dos trabalhos analisados. Assim, um aprofundamento das análises nos artigos sem referências poderá possibilitar uma reflexão a fim de auxiliar no entendimento do não uso de referências acerca do conceito.

Em uma pesquisa realizada por Boruchovitch e Bzuneck (2004) os autores apontam a limitação das opções do uso do conceito motivação no ambiente educacional. De maneira semelhante, nosso levantamento - realizado em um período complementar ao da pesquisa citada anteriormente - com os dados do ENPEC, evidenciou que são poucos os referencias que discutem acerca da motivação do aluno e são poucos os trabalhos que relacionam a motivação do aluno no ambiente educacional, sendo, portanto, necessária uma ampliação dos levantamentos, considerando os periódicos da área, para assim obtermos uma dimensão mais ampla do cenário nacional acerca do tema.

Contudo, entendemos que esta forma de leitura e interpretação é qualitativa e subjetiva. Os indícios apontados a partir da metodologia utilizada poderão auxiliar na ampliação de novas pesquisas e novos questionamentos acerca do tema motivação do aluno no Ensino de Ciências. 


\title{
The motivation in Science Education: analysis of ten years of papers presented at ENPEC
}

\begin{abstract}
This paper presents the results of an investigation about the motivation concept. It is based on papers published at the National Research Meeting on Science Education (ENPEC). For the first analytical stage, all the papers published in the annals of the last six editions of ENPEC (2005 to 2015) were analized, totalizing 5693 articles. In sequence, we conducted searches for the term motivation, resulting in 93 papers, which constituted the corpus of the present research. As a methodological tool for analysis and data collection, textual analysis was used, with emphasis on Content Analysis. From the results, it was possible to verify that: in the ENPEC the works related to the motivation concept did not represent a significant theme among the themes researched during the last six editions; the number of searches that bring the term motivation to their common sense understanding is high, approximately $56 \%$ of the total; and the papers that seek to discuss the concept of motivation in a reasoned way show a limitation of the references used, evidencing some authors, who have strong academic links.
\end{abstract}

KEYWORDS: Motivation. Content analysis. ENPEC. 


\section{REFERÊNCIAS}

BARDIN, L. Análise de conteúdo. Lisboa: Edições 70, 1977, 3.ed., 2004.

BORUCHOVITCH, E.; BZUNECK, A. J. A motivação do aluno contribuição da psicologia contemporânea. 4.ed. Rio de janeiro: Vozes, 2009.

BEZERRA, N. J. F.; SCARTAZZINI, L. S. Ensino-aprendizagem da geometria analítica: uma proposta utilizando o GPS (sistema de posicionamento global) como recurso didático e fator de motivação da aprendizagem. In: Atas do IX Encontro Nacional de Pesquisa em Educação em Ciências - V ENPEC, 2005. Disponível em: <http://abrapecnet.org.br/atas_enpec/venpec/conteudo/artigos/3/pdf/p137.pdf >. Acesso em 25 mar. 2017.

CAVENAGHI, A. R. A.; BZUNECK, J. A. A motivação de alunos adolescentes enquanto desafio na formação do professor. In: IX Congresso Nacional de Educação - EDUCERE, III Encontro Sul Brasileiro de Psicopedagogia, 2009. Disponível em: <http://www.pucpr.br/eventos/educere/educere2009/anais/pdf/1968_1189.pdf $>$ Acesso em 25 abr. 2017.

GOYA, A.; LABURÚ, C.E.; Uma estratégia de investigação multimodal para física. Atas do IX Encontro Nacional de Pesquisa em Educação em Ciências - IX ENPEC, 2013. Disponível em:

<http://abrapecnet.org.br/atas_enpec/ixenpec/atas/resumos/R0339-1.pdf> Acesso em: 11 abr. 2017.

ILLERS, K. Teorias contemporâneas da aprendizagem. Porto Alegre, 2013.

MORAES, R.; GALIAZZI, M. C. Análise Textual Discursiva. ljuí: Ed. Unijuí, 2007.

PIMENTA, Selma Garrido (Org.). Saberes pedagógicos e atividade docente. 3.ed. São Paulo: Cortez, 2002.

SETTI, B. D.; GRANDO, N. I. Contribuições das teorias da aprendizagem para a Educação Matemática: três aportes teóricos. In: XIV Conferência Interamericana de Educação Matemática. México, 2015. Anais... Disponível em: $<$ http://xiv.ciaemredumate.org/index.php/xiv_ciaem/xiv_ciaem/paper/viewFile/418/195>. Acesso em: 24 abr. 2017. 
STANZANI E. L.; GUARNIERI P. V.; BROIETTI, F. C. D.; BASTOS F. O papel da História da Ciência no Ensino de Química: ideias iniciais de futuros professores. In: Atas do IX Encontro Nacional de Pesquisa em Educação em Ciências - X ENPEC, 2015. Disponível em: < http://www.abrapecnet.org.br/enpec/xenpec/anais2015/lista_area_03.htm/r1841-1>. Acesso 25 mar. 2017.

TARDIF, Maurice. Saberes docentes e formação profissional. 4a edição. Petrópolis, RJ: Vozes, 2014.

TARDIF, Maurice; LESSARD, Claude. 0 trabalho docente: elementos para uma teoria da docência como profissão de interações humanas. 4a edição. Petrópolis, RJ: Vozes, 2008.

TODOROV, J. C.; Moreira, M. B. Conceito de Motivação na Psicologia. Revista Brasileira de Terapia Comportamental e Cognitiva, n. 1, p.119-132, 2005

VIGOTSKI, L. S. A construção do pensamento e da linguagem. São Paulo Martins Fontes, 2001.

Recebido: 15 ago. 2017

Aprovado: 15 dez. 2017

DOI: 10.3895 /actio.v2n3.6820

Como citar:

CARVALHO, W.; STANZANI, E. L.; PASSOS, M. M. A motivação no Ensino de Ciências: análise de dez anos de trabalhos apresentados no ENPEC. ACTIO, Curitiba, v. 2, n. 3, p. 97-114, out./dez. 2017. Disponível em:

<https://periodicos.utfpr.edu.br/actio>. Acesso em: XXX.

Correspondência:

Wilson Carvalho

Universidade Estadual de Londrina, Centro de Ciências Exatas, Programa de Pós Graduação em Ensino de Ciências e Educação Matemática

Rodovia Celso Garcia Cid, km 379 / PR 445. CEP: 86051-990 - Londrina, PR - Brasil, Brasil.

Direito autoral: Este artigo está licenciado sob os termos da Licença Creative Commons-Atribuição 4.0

Internacional. 